J. Gynäkol. Endokrinol. CH 2020 · 23:121-124 https://doi.org/10.1007/s41975-020-00164-x

C Der/die Autor(en) 2020

\section{Eberhard Seifert}

Abteilung Phoniatrie, Universitätsklinik für Hals-, Nasen- und Ohrenkrankheiten, Kopf- und Halschirurgie, Inselspital, Bern, Schweiz

\title{
Wie funktioniert die Stimme?
}

Im alltäglichen Umgang sprechen wir oft mühelos miteinander und setzen unsere Stimme wie selbstverständlich ein: wir tauschen Informationen aus. In unseren Kommunikationssituationen impliziert die Stimme aber gleichzeitig immer auch den Ausdruck von Gefühlen, von Stimmungen: Die Stimme macht hörbar, was im Menschen vorgeht.

Auf der einen Seite beeinträchtigt also die gestörte Stimme die zwischenmenschliche Kommunikation, auf der anderen Seite kann die gestörte Stimme aber auch Störungen der zwischenmenschlichen Kommunikation aufzeigen.

Der Ton unserer Stimme, das Stimmsignal, entsteht durch die Vibrationen der Stimmlippen, die zwischen dem Schildknorpel und den Stellknorpeln gespannt sind. Abb. 1 zeigt den laryngoskopischen Blick auf den Larynx mit den einzelnen anatomischen Strukturen in der Respirationsphase: Die Stimmlippen sind abduziert. Bei der Phonation adduzieren die Stimmlippen und im Idealfall kommt es $\mathrm{zu}$ einem vollständigen Stimmlippenschluss.

Der komplexe Aufbau der Stimmlippe mit M. vocalis, Lig. vocale (Stimmband), Lamina propria (Reinke'scher Raum) und oberflächlicher Schleimhaut ermöglicht, dass die adduzierten Stimmlippen durch den bei der Exspiration entstehenden Atemstrom in Schwingungen gebracht werden. Durch die Verschiebung der oberflächlichen Schleimhaut auf der Lamina propria entsteht die wellenförmige Bewegung der Stimmlippenoberfläche, die sogenannte Randkantenverschiebung in einer dreidimensionalen Ausdehnung. Daraus resultiert schliesslich ein Ton. Die normale mittlere Sprechstimmlage liegt bei der männlichen Stimme bei etwa $100 \mathrm{~Hz}$, das heisst die
Stimmlippen schwingen $100 \mathrm{x} / \mathrm{s}$, bei der weiblichen Stimme bei ca. $200 \mathrm{~Hz}$.

Die jeweilige Tonhöhe ist abhängig von der Masse der Stimmlippen, von der Spannung und vom subglottischen Druck. Je mehr Masse die Stimmlippen haben, desto tiefer ist der Ton, je mehr Spannung sie aufweisen und je höher der subglottische Druck ist, desto höher wird der Ton. Das zweite Charakteristikum eines Tones ist die Lautstärke. Hier gilt, je höher der subglottische Druck ist und je schneller die Luft strömt, desto lauter ist der Ton.

Für die Steuerung der Nuancen der Stimmgebung sind die sechs inneren und der äussere Kehlkopfmuskel verantwortlich. Die innere Muskulatur wird durch den N. laryngeus inferior oder recurrens, der äussere Kehlkopfmuskel durch den N. laryngeus superior innerviert, die beide aus dem Nervus vagus abgehen.

Das so gebildete Tonsignal wird durch den Rachen, den Pharynx, die nasalen Räume und den Mund nach aussen geleitet. Diese Strukturen werden auch als Resonanzraum bezeichnet. Durch Veränderungen des Resonanzraumes werden unterschiedliche Obertöne, die Formanten, gebildet und dadurch entstehen die unterschiedlichen Sprachlaute [1]. Dieses komplexe Geschehen kann man an einem einfachen Beispiel demonstrieren: Spricht man ein langes /i/ und spitzt im Verlauf die Lippen, verlängert sich der Artikulationstrakt. Damit verändern sich die Obertöne des Tonsignals und aus dem /i/ wird ein / ̈̈/.

\section{Entwicklung der Stimme}

Der erste Laut eines Menschen, der Neugeborenenschrei, hat eine Grundfrequenz von $440 \mathrm{~Hz}$, liegt also bei Kammerton a1. Mit dem zunehmenden Kör- perwachstum wird der Ton tiefer und der Stimmumfang grösser. In der Pubertät kommt es durch den Androgeneinfluss $\mathrm{zu}$ einem starken Wachstumsschub des Kehlkopfes: Bei den Jungen verlängern sich die Stimmlippen um $1 \mathrm{~cm}$, bei den Mädchen um 3-4mm. Dadurch sinkt die Grundfrequenz bei den Jungen um eine Oktave, bei den Mädchen um eine Terz. Nicht selten verläuft das Wachstum unkoordiniert und die Stimme wird besonders sensibel. Wenn dann zusätzlich noch psychosoziale Belastungssituationen beim Erwachsenwerden auftreten, kann eine Mutationsstimmstörung resultieren, der verlängerte „Stimmbruch“: Die Stimme kippt zwischen der Kopfund Bruststimme hin und her. Ist der Stimmwechsel einmal geschafft, bleibt die Stimme beim Mann meist stabil, bis im höheren Alter die physiologischen Alterungsprozesse zu einer Presbyphonie, der Altersstimme, führen können: Die Stimme kann schwächer, brüchiger und höher werden.

Bei der Frau können sich die physiologischen hormonellen Veränderungen auch auf die Stimme auswirken, denn die Stimmlippenschleimhaut reagiert auf die Geschlechtshormone ähnlich wie das Vaginalepithel. Vor der Menstruation ermüdet die Stimme rascher, die Stimme wird kraftloser. Während der Schwangerschaft können die Stimmlippen leicht anschwellen, die Stimme wird wärmer, kräftiger, oft bleibt dies nach der Geburt des Kindes bestehen. In der Menopause wird die Stimme tiefer, manchmal kraftloser und die Resonanz nimmt ab. Im höheren Lebensalter kann sich dann auch hier die Presbyphonie entwickeln. 


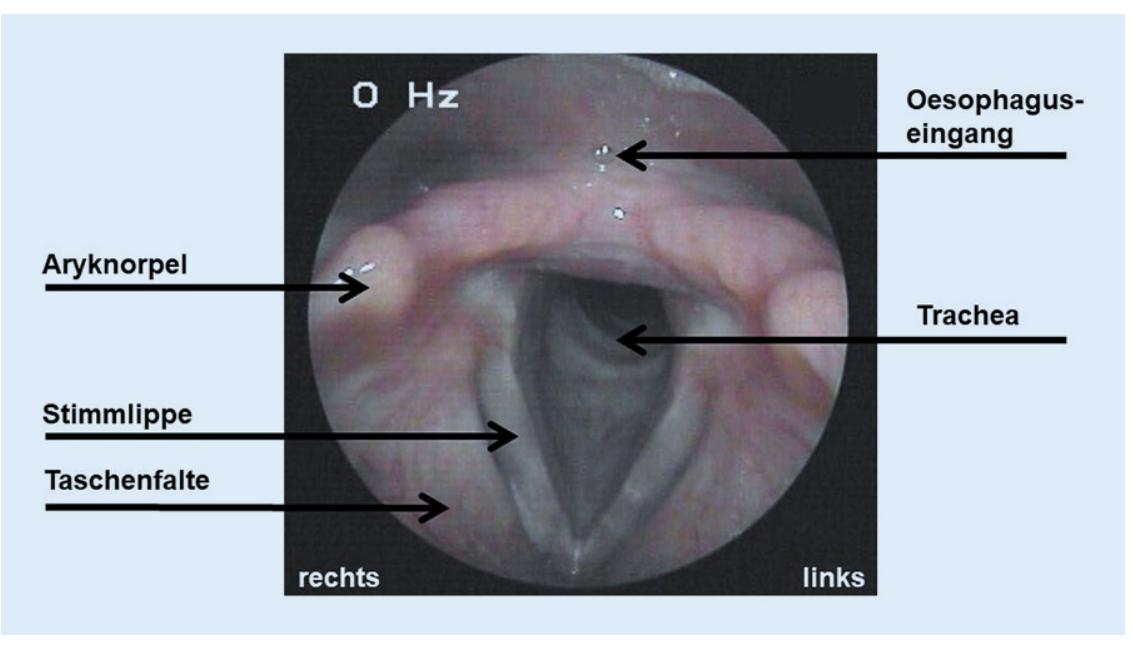

Abb. 1 A Laryngoskopisches Bild des Kehlkopfs

\section{Stimmstörung}

Eine Stimmstörung (Dysphonie) wird definiert durch:

- einen eingeschränkten Stimmklang und/oder

- eine verminderte stimmliche Leistungsfähigkeit und/oder

- laryngeale Missempfindungen („Klossgefühl“ im Hals)

Es wird zwischen einer organischen und einer nicht-organischen Stimmstörung unterschieden. Organische Stimmstörungen beruhen z. B. auf Missbildungen des Kehlkopfes, Entzündungen, Verletzungen, Lähmungen oder gutartigen oder bösartigen Tumoren. Nicht-organische Stimmstörungen, auch „funktionell“ genannt, werden definiert als Dysphonie ohne verursachende organische Pathologie. Es können sich aber in der Folge organische Veränderungen bilden, wie zum Beispiel Stimmlippenverdickungen, Knötchen, Polypen oder auch das Kontaktgranulom.

Bei den funktionellen Stimmstörungen spielen immer auch psychosoziale Faktoren eine Rolle [2]. Im Extremfall kann eine psychogene Aphonie resultieren, dem Menschen ,verschlägt es die Stimme“. Diese Patienten können dann trotz regelrechter Anatomie und Funktionsfähigkeit des Larynx nur flüstern.

\section{Diagnostik}

Die europäische laryngologische Gesellschaft empfiehlt eine multidimensionale Herangehensweise in der Diagnostik [3]. Dazu gehört die subjektive Bewertung der Stimmstörung durch die Patienten selbst mittels standardisiertem Fragebogen (z.B. Voice Handicap Index, VHI), eine perzeptive Einschätzung der Stimme durch die Untersuchenden, eine videostroboskopische Darstellung der schwingenden Stimmlippen, eine akustische Stimmschallanalyse sowie aerodynamische Parameter, wie z.B. die mittlere Phonationsdauer. Nicht zu vergessen ist die ausführliche Anamnese am Anfang der Diagnostik auch im Hinblick auf psychosoziale Risikofaktoren. Denn diese lassen sich erst im Verlauf eines Gespräches eruieren. Die Zusammenschau aller dieser Parameter bieten den Schlüssel zur korrekten Diagnosestellung und ermöglichen eine adäquate Therapie.

Zwei Verfahren sollen etwas detaillierter beschrieben werden:

- Die Laryngostroboskopie beruht auf dem Prinzip, dass die schnell ablaufenden periodischen Stimmlippenschwingungen durch eine Blitzlichtquelle mit einer geringen Zeitverzögerung beleuchtet werden. Dadurch entsteht für die Untersuchenden der Eindruck wie eine Zeitlupendarstellung. Die Stimmlippenfunktion kann beurteilt werden, der Schwingungsablauf wird dargestellt und auch kleinste Veränderungen lassen sich dadurch sehr gut erkennen.

- In der akustischen Stimmschallanalyse wird die mittlere Sprechstimmlage in drei verschiedenen Lautstärken gemessen. Es wird die Rufstimme ermittelt und der Stimmumfang und die Stimmdynamik der Singstimme. Auch objektive Parameter der Stimme wie Jitter, Shimmer, Noise to Harmonic Ratio können bestimmt werden. Mit dem Dysphonia Severity Index (DSI) kann die Ausprägung der Dysphonie quantifiziert werden.

\section{Therapeutische Möglichkeiten}

Medikamentöse Therapien kommen bei akuten und/oder chronischen Entzündungen zum Einsatz. Eine akute Laryngitis wird symptomatisch, antiphlogistisch behandelt. Wichtig ist hier, die Stimme zu schonen und nicht zu überlasten. Bei einer chronischen Laryngitis wird häufig eine Inhalationstherapie mit corticoidhaltigen Lösungen verwendet. Liegen Zeichen für eine Refluxlaryngitis vor, ist eine ausreichend hoch dosierte PPITherapie $(2 \times 40 \mathrm{mg})$ unter Zuhilfenahme eines Alginats nach dem Essen für einen Zeitraum von mindestens 6 Wochen indiziert.

Die Phonochirurgie hat eine Funktionsverbesserung der Stimme zum Ziel. Phonochirurgische Massnahmen kommen vor allem bei gutartigen Veränderungen der Stimmlippen, wie Polypen, Zysten oder Papillomen zum Einsatz. Bei einseitigen Stimmlippenlähmungen mit resultierendem unvollständigem Stimmlippenschluss kann eine Unterfütterung der gelähmten Stimmlippe (=Augmentation) mit z.B. körpereigenem Fett, Hyaluronsäure, Hydroxylapatit oder Silikon vorgenommen werden.

Mit einer logopädischen Stimmtherapie werden funktionelle Stimmstörungen angegangen: Durch das Arbeiten an der Atmung wird die Basis für eine gute Stimmfunktion gelegt. Die Koordination zwischen Respiration-PhonationArtikulation wird optimiert. Durch verschiedene, individuell angepasste Übungen soll ein Abbau des übermässigen und Aufbau eines adäquaten Tonus der Atmung, der Larynxmuskulatur, der 
Stimmlippen, schliesslich des ganzen Individuums erreicht werden. Durch regelmässiges Üben der erlernten Technik erleben die Patienten eine Verbesserung und Stabilisierung ihrer Stimmfunktion.

Bei der Behandlung einer Dysphonie dürfen auch die psychosozialen Aspekte nicht ausgeklammert werden. Wenn im therapeutischen Setting die Stimmung mit einbezogen wird, kann eine gute Stimme aufgebaut werden. Nicht selten ist hier dann auch eine psychologische Unterstützung der PatientInnen indiziert.

So kann durch die Berücksichtigung des bio-psycho-sozialen Ansatzes das Ziel einer nachhaltigen Stimmtherapie erreicht werden: Mensch und Stimme sind im Einklang.

\section{Korrespondenzadresse}

\section{Prof. Eberhard Seifert}

Abteilung Phoniatrie, Universitätsklinik für Hals-, Nasen- und Ohrenkrankheiten, Kopfund Halschirurgie, Inselspital 3010 Bern, Schweiz

Eberhard.Seifert@insel.ch

\section{Prof. Eberhard Seifert Leitender Arzt}

Funding. Open access funding provided by University of Bern

\section{Einhaltung ethischer Richtlinien}

Interessenkonflikt. E. Seifert gibt an, dass kein Interessenkonflikt besteht

Für diesen Beitrag wurden von den Autoren keine Studien an Menschen oder Tieren durchgeführt. Für die aufgeführten Studien gelten die jeweils dort angegebenen ethischen Richtlinien.

Open Access. Dieser Artikel wird unter der Creative Commons Namensnennung 4.0 International Lizenz veröffentlicht, welche die Nutzung, Vervielfältigung, Bearbeitung, Verbreitung und Wiedergabe in jeglichem Medium und Format erlaubt, sofern Sie den/die ursprünglichen Autor(en) und die Quelle ordnungsgemäß nennen, einen Link zur Creative Commons Lizenz beifügen und angeben, ob Änderungen vorgenommen wurden.

Die in diesem Artikel enthaltenen Bilder und sonstiges Drittmaterial unterliegen ebenfalls der genannten Creative Commons Lizenz, sofern sich aus der Abbildungslegende nichts anderes ergibt. Sofern das betreffende Material nicht unter der genannten Creative Commons Lizenz steht und die betreffende Handlung nicht nach gesetzlichen Vorschriften erlaubt ist, ist für die oben aufgeführten Weiterverwendungen des $\mathrm{Ma}$ terials die Einwilligung des jeweiligen Rechteinhabers einzuholen.
Weitere Details zur Lizenz entnehmen Sie bitte der Lizenzinformation auf http://creativecommons.org/ licenses/by/4.0/deed.de.

\section{Literatur}

1. Richter B (2014) Die Stimme. Henschel-Verlag, Leipzig

2. Seifert E, Kollbrunner J (2005) Stress and distress in non-organic voice disorders. Schweiz Med Wochenschr 135:387-397

3. Dejonckere PH, Bradley P, Clemente P, Cornut G, Crevier-Buchmann L, Friedrich $G$, Van den Heyning P, Remacle M, Woisard V (2001) A basic protocol for functional assessment of voice pathology, especially for investigating the efficacy of (phonosurgical) treatments and evaluating new assessment techniques. Guideline elaborated by the Committee on Phoniatrics of the European Laryngological Society (ELS). Eur Arch Otorhinolaryngol 258:77-82

Hinweis des Verlags. Der Verlag bleibt in Hinblick auf geografische Zuordnungen und Gebietsbezeichnungen in veröffentlichten Karten und Institutsadressen neutral.

\section{Drogenkonsum der Mutter kann bei Kindern Diabetes auslösen}

Zahlen aus den USA geben an, dass 5 bis 10 $\%$ aller Mütter im Laufe ihrer Schwangerschaft Psychostimulanzien konsumieren. Neben negativen Effekten auf das Gehirn entwickeln viele Neugeborene eine Störung des Blutzuckerhaushaltes, wovon statistisch gesehen mehr weibliche Babys betroffen sind. Die Studie, die auf Beobachtungen im Menschen beruht und in Mausmodellen im Detail durchgeführt wurde, ging der Frage nach, ob die zugrundeliegenden Mechanismen für diese Stoffwechsel-Defekte ähnlich jenen im Gehirn sind. Die Forscher untersuchten dafür die Konsequenzen der Psychostimulanzien auf die Entwicklung der Beta-Zellen und maßen die lebenslangen Auswirkungen auf die Insulinproduktion. Im fetalen Gehirn benutzen diese Drogen DopaminSignalwege, die es im sich entwickelnden Pankreas nicht gibt. Stattdessen beeinflussen sie im Pankreas mittels des SerotoninTransporters Serotonin-Signalwege und verändern so die Entwicklung der BetaZellen. „Die Beta-Zellen im Pankreas haben durch die Drogen Modifikationen ihrer epigenetischen Programme erfahren, wodurch sich die Identität dieser Zellen auf eine Weise ändert, dass die Ausschüttung des Hormons Insulin beeinträchtigt wird", erklärt Studienleiter Tibor Harkany, , dieses Defizit beruht auf der epigenetisch veränderten Programmierung als Folge des Psychostimulanzien-Konsums der Mutter und bleibt über das gesamte Leben des Kindes bestehen." Denn selbst bei unregelmäßigem Drogenkonsum - im Maus-Versuch wurden nur an 3 Schwangerschaftstagen Drogen verabreicht - ergab der Glukosetoleranztest beim Nachwuchs auch noch im Erwachsenenalter abnorme Blutzuckerwerte. Auch hier zeigte sich der bereits beim Menschen beobachtete Geschlechterunterschied - weiblicher Nachwuchs war stärker betroffen. „Diese in unserem präklinischen Versuch entschlüsselten Mechanismen geben deutliche Hinweise auf die Wirkungsweise von Drogen, die auch im menschlichen Organismus bedeutsam sind", erklärt Harkany.

Quelle: www.meduniwien.ac.at 
Hier steht eine Anzeige.

\section{曾 Springer}

